\title{
Non-Intrusive Ice Accretion Detection and Measurement System
}

\author{
Ricardo M. U. Entz ${ }^{1}$, Raphael de Andrade Jorge ${ }^{2}$ \\ 1 Airbus Group Innovations, 81663 Munich, Germany \\ ricardo.entz@airbus.com \\ 2 University of Sao Paulo, Engineering School of Sao Carlos
}

\begin{abstract}
Precisely detecting and measuring accreted ice on aircraft requires the overcoming technical challenges posed by the harsh environment outside the aircraft pressure vessel. This work describes a novel ice detection and measurement system based on the principle of light spatial spreading [1]. The main advantages of this method are its robustness and the capability of measuring the target surface directly. Initial results on a proof of concept implementation show that the measurement principle is valid and can also be applied for thickness measurement.
\end{abstract}

\section{Key words}

aircraft ice measurement, light spatial spreading, image processing, optical properties of ice, multiple scattering

\section{Introduction}

In-situ measurement of ice accretion is a subject that has been overlooked in flight testing for its difficulty both in the technical and the theoretical aspects. Current icing tests employ indirect measurements such as monitoring of ice accretion on a standard cylinder and recording of atmospheric conditions. While these methods are simple and reliable, they do not substitute for ice accretion measurement on the target surfaces. Test bodies are subject to different flow conditions leading to discrepancies in collection efficiency and in accretion rate[2]. Current methods are especially limited in the development of new anti-ice systems; their validation requires precise detection and measurement of ice accretion where aircraft safety and performance are most affected wings, elevators and rotors.

This work describes a non-intrusive ice accretion measurement system exploiting the phenomenon of spatial spreading of a light beam [1]. The propagation of light in ice is used for ice thickness measurement by Gagnon [3] and Ikiades et al. [4]. Gagnon assumes refraction as the source of spatial spreading, and measures total internal reflection to determine ice layer thickness. His hypothesis assumes in practice a planar, transparent ice layer. Ikiades et al., on the other hand, assume spatial spreading of the light beam from multiple scattering events, but their implementation requires the installation of optical fibres through the wind leading edge and the placement of sensors parallel to the wing spanwise direction. Such limitations de- mand costly and time consuming modifications if the system is to be applied in flight testing.

Our implementation can be described as a hybrid of both approaches, taking the non-intrusive installation of Gagnon but using spatial spreading as the measurement principle.

\section{Ice accretion}

Accreted ice is divided in two categories: rime ice and clear ice.

Rime ice is formed in conditions where supercooled water droplets freeze rapidly, trapping air bubbles in the ice matrix. The bubbles act as light scatterers and causes rime ice to have a white and opaque appearance. Clear ice, on the other hand, is transparent and homogeneous; it is formed in temperatures near the water freezing point, being the result of slower freezing process allowing air bubbles not to be trapped in the ice matrix $[5,6,7,8]$.

Between pure clear and rime ice there are infinitely many possible variations, with relative densities ranging from 0.92 (ice density) to less than 0.4 [9]. There is no clear cut classification to determine whether ice is clear or rime, leaving room for an intermediate category denominated mixed ice.

This work will present results for rime ice, except where indicated, as the general spreading behaviour is similar, and the remaining conclusions of feasibility and technical challenges are 
the same for both kinds of accretion.

\section{Spatial spreading}

Light travelling in non-homogeneous environments is subject to random scattering events, which combine reflection and refraction. The phenomenon can be represented by microscopic models which account for each scattering event in the ice [10]. Their application requires data on ice micro structure, namely size, shape and location of each bubble in the ice; the lack of detailed information precludes the application of microscopic models in our analysis.

On the other end of the model spectrum, macroscopic models based on the radiative transfer equation are capable of predicting some of the spreading behaviour based on average properties of the scattering medium[11]. A major application of such models is in the field of computer graphics, namely for rendering of clouds, smoke or milk.

One example of macroscopic formulation is proposed by Tessendorf $[12,13]$. In his model, multiple scattering is assumed to be a stochastic process where light has a probability of being scattered (deflected) by an average (RMS) angle $\left\langle\theta^{2}\right\rangle$. In cases where $\left\langle\theta^{2}\right\rangle$ is sufficiently small, an analytic point spread function (PSF) is found:

$$
I(\vec{r}, \vec{n}) \propto \exp \left(-\frac{(\vec{r}-\vec{n} I R(q))^{2}}{2\left\langle\theta^{2}\right\rangle b^{3} h(q)}\right)
$$

Where:

$I(\vec{r}, \vec{n})$ is the resulting radiance profile;

$\vec{r}$ and $\vec{n}$ are the direction vectors perpendicular and parallel to the light propagation;

$a$ and $b$ are the absorption and scattering coefficients;

$I=\left(a b\left\langle\theta^{2}\right\rangle\right)^{-1 / 2}$ is a characteristic length relating scattering and absorption phenomena;

$q$ is the propagation length (ice thickness) divided by $l$;

$$
\begin{aligned}
& R(x)=(\cosh (x)-1) \sinh ^{-1}(x) ; \text { and } \\
& h(x)=(x \sinh (x)+2(1-\cosh (x))) \sinh ^{-1}(x) .
\end{aligned}
$$

The coefficients $a$ and $b$ can be estimated using the Mie theory[14, 15, 16].

In the following sections this model will be compared with the obtained results.

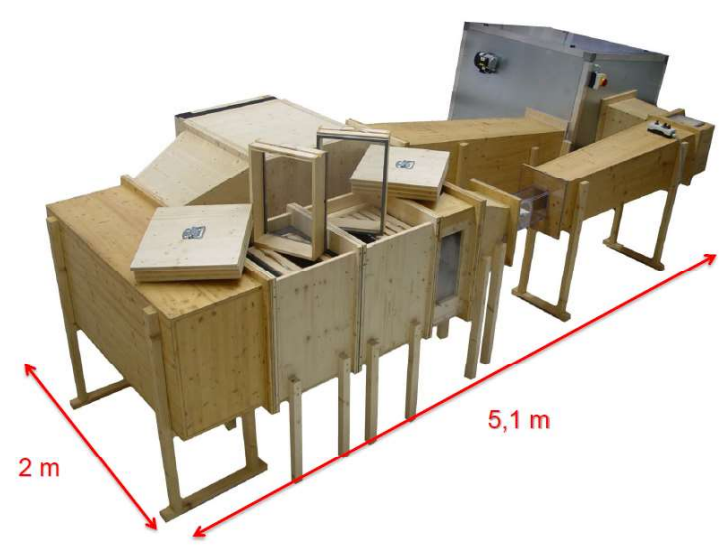

Figure 1: iCORE wind tunnel. Test section dimensions: $150 \times 100 \times 450 \mathrm{~mm}^{3}$

\section{Proof of concept measurement}

In order to confirm that the physical phenomenon is measurable, a proof of concept experiment was executed.

The test campaign was carried out in the iCORE (Icing and Contamination Research Facility) wind tunnel (Fig. 1)[17]; it is capable of reaching a free stream temperature down to $250 \mathrm{~K}$ at $M 0.45$. Water droplets with diameters in the range $10-25 \mu \mathrm{m}$ are created and injected in the free stream before the test section. There is full optical access in the test section, making iCORE ideal for the implementation of our measurement system.

The test model was a NACA0012 wing equipped with a removable insert used for direct measurement of accreted ice and the testing of different ice phobic coatings (cf. Fig. 2). For the reference measurement, instead of manually measuring the ice thickness, we placed a camera along the spanwise direction of the wing. This camera provided direct measurement of the ice accretion; for reference transparent, graduated side plates (baffles) were used (Fig. 3).

The measurement of the spatial spreading was performed by projecting a laser line perpendicular to the wing spanwise direction. The images were recorded with a camera placed on the same plane as the laser sheet, as shown in the diagram of Fig. 2. The spreading phenomenon observed with this configuration is illustrated in Fig. 4.

The acquired images were corrected for camera distortions and projection error, then the intensity profile of the laser line was sampled at several cross sections. 


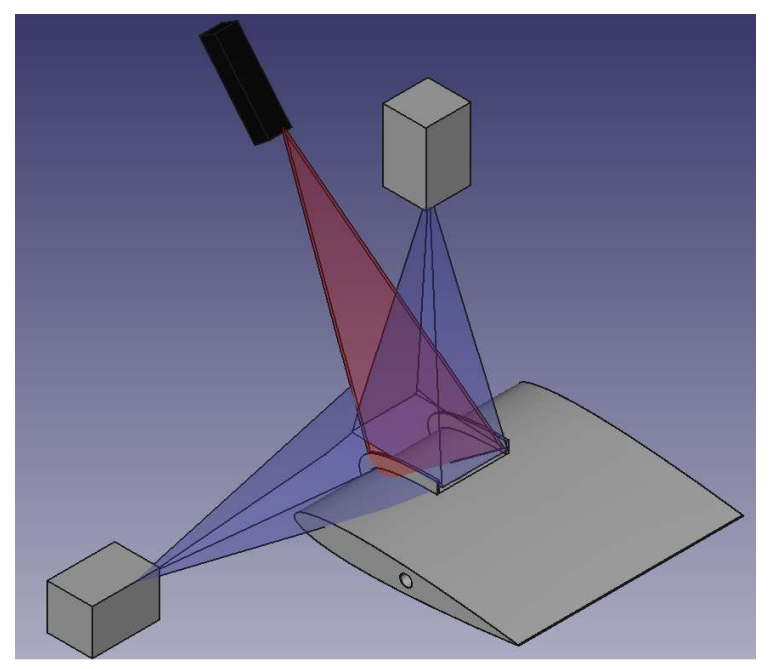

Figure 2: Full experimental set up, including wing with insert, two cameras (parallel to the wing chord for direct ice measurement and perpendicular for beam spreading measurement), and laser sheet (red plane).

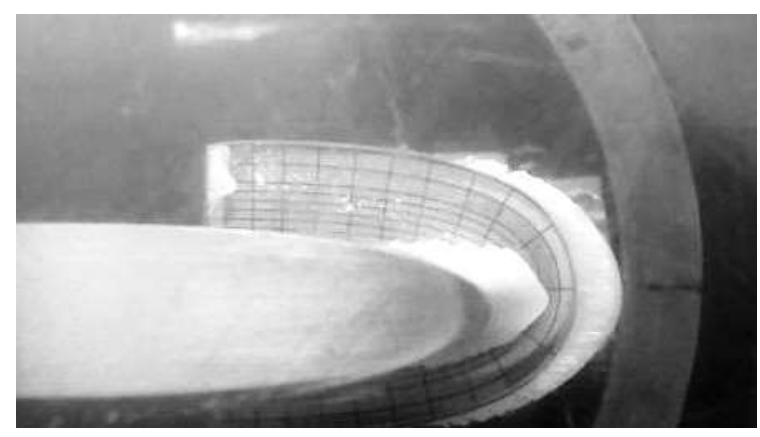

Figure 3: Side camera view with graduated baffle.

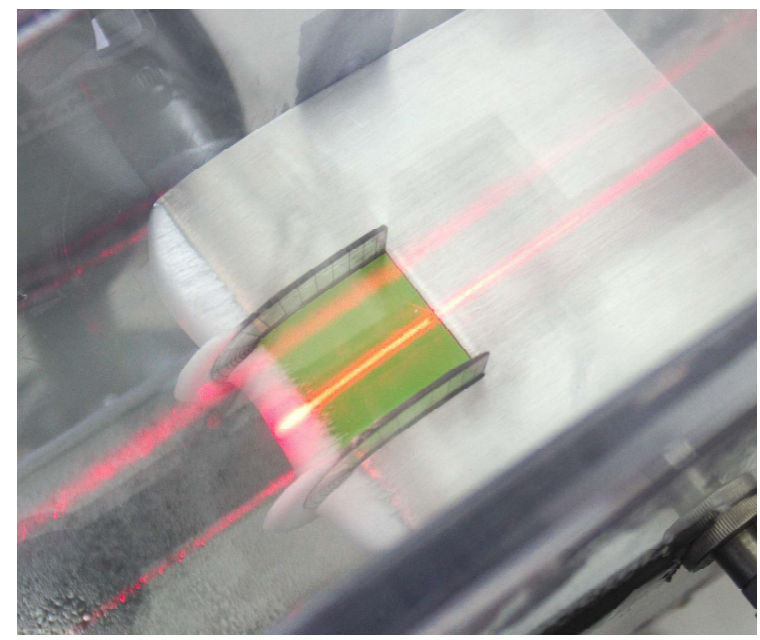

Figure 4: Visualization of the spatial spreading phenomenon on rime ice.
The sampled profiles were normalized to the range $0 \leq I(x) \leq 1$. Following the methodology of spreading measurement proposed by Premože [18], we assumed that the sections had an approximately Gaussian profile reducing the measurement of the line width to a curve fitting problem using the following parametric equation:

$$
I(x)=\exp \left(-\left(\frac{x-\bar{x}}{\sigma \sqrt{2}}\right)^{2}\right)
$$

Where the values of $\bar{x}$ and $\sigma$ were adjusted to obtain the best fitting profile to the measured laser intensities. The value of the coefficient $\sigma$ is then used to determine the full width at half maximum $(F W H M)$ of the profile using the following formula:

$$
F W H M=2 \sigma \sqrt{2 \ln 2}
$$

The image processing steps are illustrated in Fig. 5.

\section{Initial results}

Initial results show that the spreading phenomenon is measurable using image processing techniques. Fig. 6 shows a time series of the measured laser line width. This series starts with an increase in measured width corresponding to the beginning of the water injection. In two intervals, between images 500-1000 and between images 1500-2000 the water injection was interrupted for the measurement of constant thickness values.

The actual ice thickness could not be measured with the reference camera due to problems in the measurement methodology, namely:

Wing curvature - As the wing chord was small $(100 \mathrm{~mm})$, the leading edge curvature posed a challenge for the positioning of the laser, as most configurations had a specular reflection point which presented different spreading behaviour as other regions, as shown in Fig. 6 .

Erosion of accreted ice - The measurement of stabilized values (without water injection) could not be performed due to erosion of the ice layer by the wind.

Reference camera parallax error - The side camera had to be positioned near the wind tunnel (ca. $200 \mathrm{~mm}$ ) because of limitations with its optics and resolution. This prevented reliable measurement of the accreted ice layer because of parallax error. 

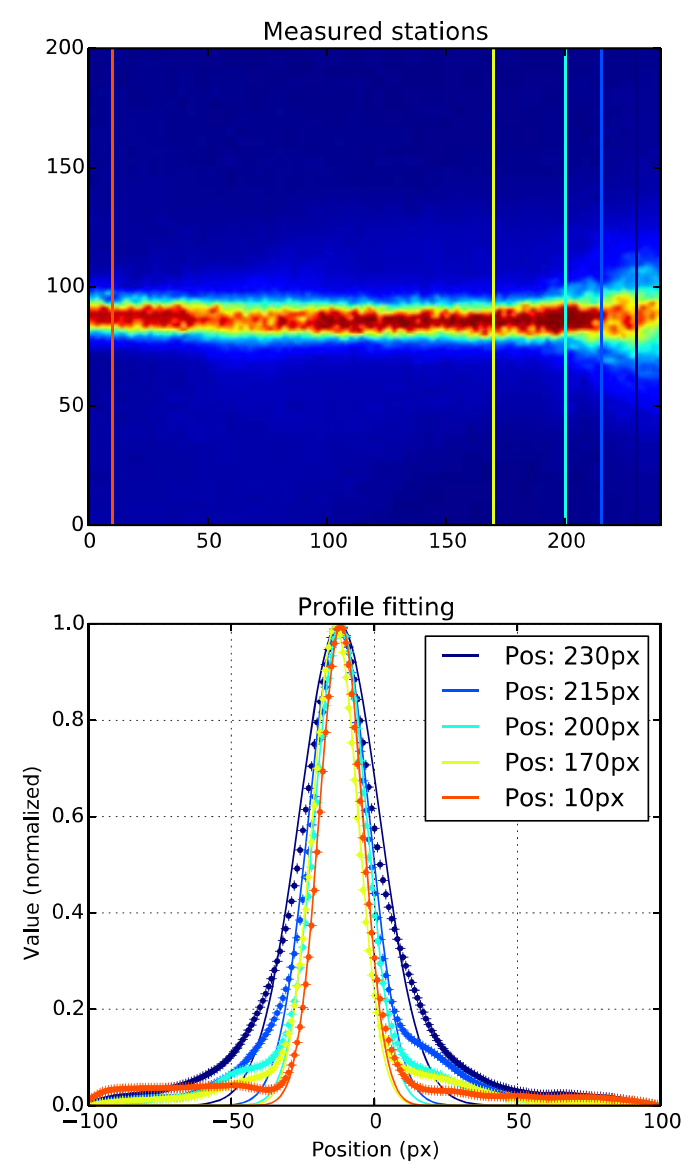

Figure 5: Visualization of the profile fitting methodology. Upper figure shows the laser line with the selected cross-sections for measurement. Lower figure shows normalized intensity profiles (dots) and fitting profiles (lines).

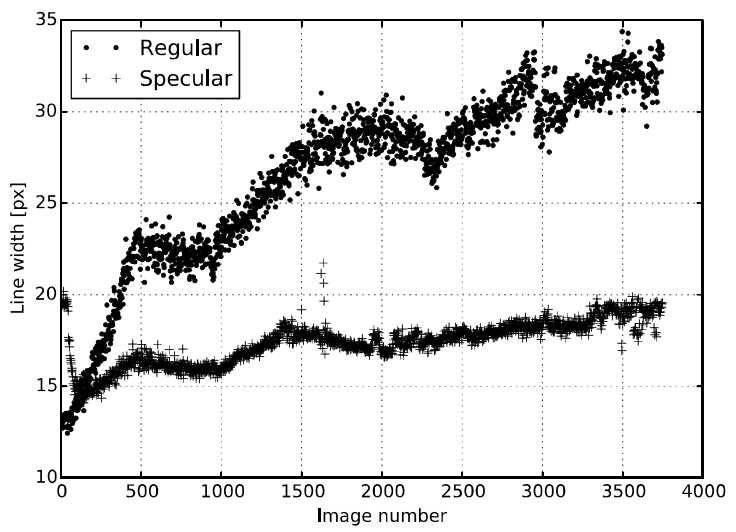

Figure 6: Raw result of first measurements. Results are presented for a regular measurement station (solid dots) and a station on the specular reflection caused by the wing curvature (open dots).

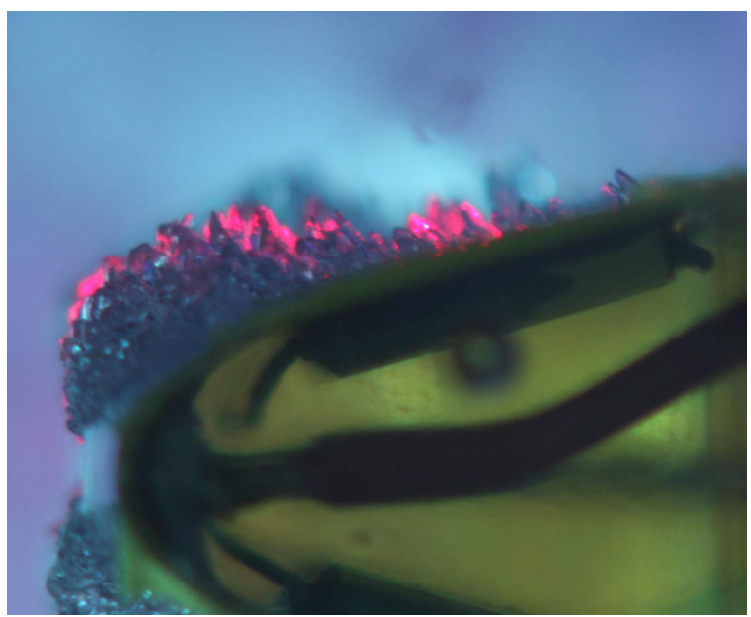

Figure 7: Image of side camera after improvement of experimental setup (cf. Fig. 3 for original images). Clear ice, original resolution $3000 \times 2000 p x^{2}$. The lack of accreted ice on the leading edge is due to the anti-ice system proposed by Strobl[19].

\section{Improvement of the experimental setup}

In order to improve the results quality a series of changes was adopted for the second experiment. First, a larger wing with chord of $250 \mathrm{~mm}$ was used. By correctly choosing the angle of attack, it was possible to have the accretion in a region with less curvature than the leading edge of the previous wing. This gave more flexibility to the positioning the laser-camera group, and also reduced the interference of curvature in the measurements.

The second modification affected the reference camera. A digital single lens reflex (DSLR) camera was used with a long focal length lens $(f=$ $432 \mathrm{~mm}$ in $35 \mathrm{~mm}$ equivalent) to allow the positioning of the camera far from the wind tunnel $(>1.5 \mathrm{~m})$. This configuration was denominated quasi-telecentric, and it reduced parallax error to less than $0.1 \mathrm{~mm}$ in the measurement range.

The improvement on the reference images allowed better observation of the accretion structure and a direct measurement with estimated uncertainty of less than $0.5 \mathrm{~mm}$. Fig. 7 shows an example of the improved image quality.

In subsequent measurements we made no attempt to measure stabilized ice shapes, as the erosion phenomenon could not be controlled in the icing wind tunnel.

\section{Results of second measurement series}

The improvements in the second measurement 

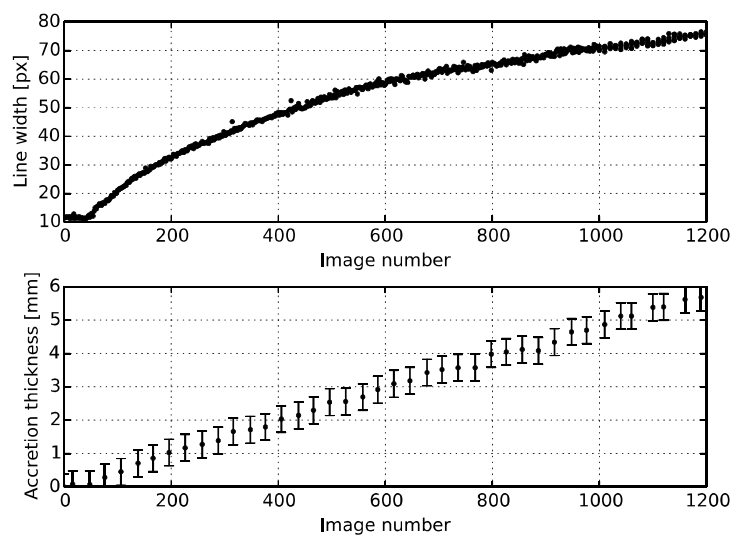

Figure 8: Second measurement results. Maximum spreading width is ca. $10 \mathrm{~mm}$.

series allowed comparison between light spreading and ice thickness results, as shown in Fig. 8. Light spreading and ice thickness are strongly correlated, with a Pearson product-moment correlation coefficient of 0.96 . However, we found that the relation between variables is non-linear.

\section{Comparison with theory}

We assume ice as a solid water matrix with spherical air bubbles, as proposed by Carras and Macklin[6]. This approximation allows the direct application of the Mie theory for the determination of the absorption and scattering coefficients needed for the application of the theoretical model.

Choosing a bubble diameter of $5 \mu \mathrm{m}$ in rime ice with density $\rho=400 \mathrm{~kg} / \mathrm{m}^{3}$ the following values are obtained:

$$
\begin{aligned}
& a=0.047 \mathrm{~m}^{-1} \\
& b=3.11 \times 10^{5} \mathrm{~m}^{-1} \\
& \left\langle\theta^{2}\right\rangle=0.31 \\
& I=\left(a b\left\langle\theta^{2}\right\rangle\right)^{-1 / 2}=0.015 \mathrm{~m}
\end{aligned}
$$

Fig. 9 shows the predicted intensity profiles and the measured width for a $1.2 \mathrm{~mm}$ wide laser beam as input. There is no qualitative nor quantitative agreement between the calculated and measured behaviour (cf. Figs. 9 and 8). The predicted width for a $5 \mathrm{~mm}$ ice layer is around $100 \mathrm{~mm}$, an order of magnitude off the measured value. In addition, the curve trend is not the same.

We found that the general behaviour predicted by theory does not change appreciably by varying ice density and bubble mean diameter within
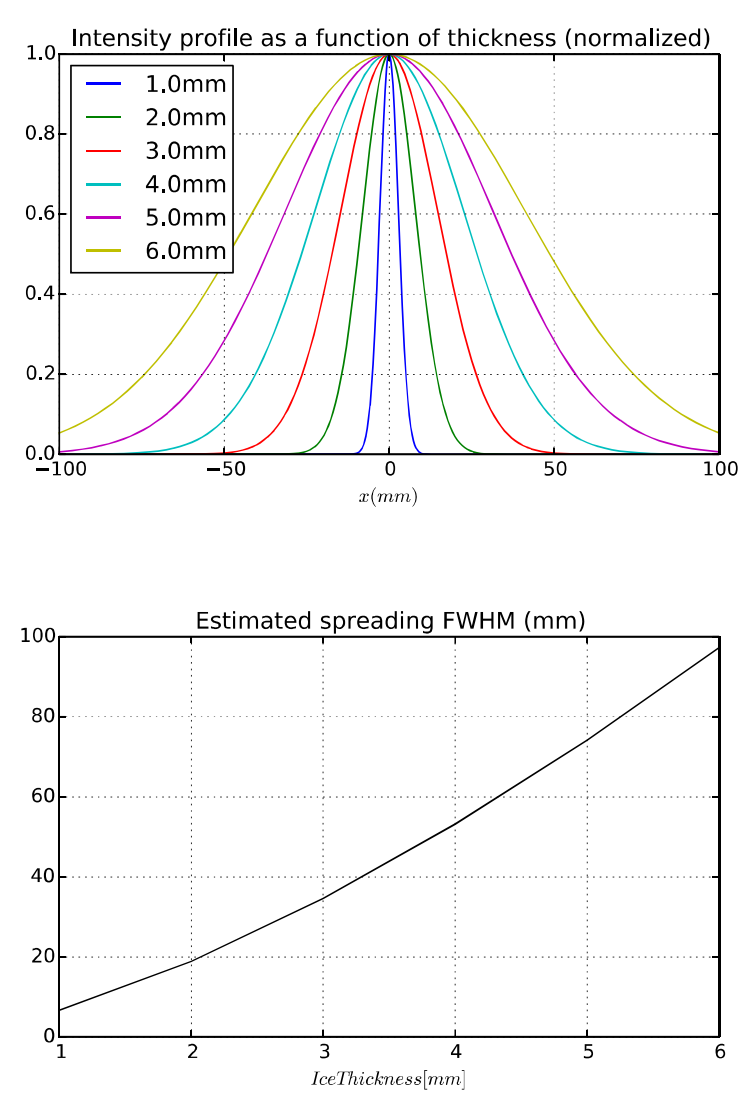

Figure 9: Resulting intensity profiles and FWHM widths as predicted by the theory.

expected values for ice. This indicates that other effects dominate the spreading in this setup.

\section{Application}

Despite the disagreement between theory and measurements, the results showed to be repeatable for similar ice types. This allowed us to create a calibration table to determine the ice thickness from light spatial spreading. The system was successfully applied in a measurement campaign in the iCORE facility $[19,20,21]$.

\section{Conclusion}

This paper presented a novel system for ice accretion measurement. Its main advantage is its simplicity, allowing its implementation using lowcost equipment.

It was shown that measurement using image processing techniques produces measurable results starting from less than $1 \mathrm{~mm}$ in ice thickness. The available theory, however, does not 
predict the measured behaviour, requiring an empirical calibration for each ice type.

The system, as described in this paper, was applied successfully for measurement campaigns in the iCORE facility. For application in other fields, however, it requires further theoretical development, notably for the extension of the measurement range without the need for exhaustive parameter sweep calibration.

\section{References}

[1] F. Olchewsky, M. Weber, and R. M. U. Entz, "Système et procédé de mesure de l'épaisseur de givre sur une surface, notamment d'aéronef, à l'aide d'un motif lumineux généré par diffusion dans le givre," 2014 (patent to be published).

[2] C. S. Bidwell and M. Papadakis, "Collection efficiency and ice accretion characteristics of two full scale and one $1 / 4$ scale business jet horizontal tails," in SAE General Aviation Technology Conference and Exposition sponsored by the Society of Automotive Engineers, 2000.

[3] R. E. Gagnon, "Method and apparatus for remote detection and thickness measurement of ice or liquid layer," 1994.

[4] A. Ikiades, G. Howard, D. J. Armstrong, M. Konstantaki, and S. Crossley, "Measurement of optical diffusion properties of ice for direct detection ice accretion sensors," Sensors and Actuators, A: Physical, vol. 140, pp. 24-31, October 2007.

[5] R. Gent, N. Dart, and J. Cansdale, "Aircraft icing," Philosophical Transactions of the Royal Society of London. Series A: Mathematical, Physical and Engineering Sciences, vol. 358, no. 1776, pp. 2873-2911, 2000.

[6] J. N. Carras and W. C. Macklin, "Air bubbles in accreted ice," Quarterly Journal of Royal Meteorological Society, vol. 101, pp. 127146, 1975.

[7] A. Heinrich, R. Ross, G. Zumwalt, J. Provorse, V. Padmanabhan, J. Thompson, and J. Riley, Aircraft Icing Handbook, vol. 1. Federal Aviation Administration, 1991.

[8] T. Ratvasky, B. Barnhart, and S. Lee, "Current methods for modeling and simulating icing effects on aircraft performance, stability and control," in AIAA Atmospheric Flight Mechanics Conference and Exhibit, 2008.

[9] W. C. Macklin, "The density and structure of ice formed by accretion," Quarterly Journal of the Royal Meteorological Society, vol. 88, pp. 30-50, 1962.

[10] W. R. C. Somerville, B. Augui, and E. C. L. $\mathrm{Ru}$, "Accurate and convergent T-matrix calculations of light scattering by spheroids," Journal of Quantitative Spectroscopy \& Radiative Transfer, vol. 160, pp. 29-35, 2015.

[11] C. F. Bohren, "Multiple scattering of light and some of its observable consequences," Am. J. Phys, vol. 55, no. 6, pp. 524-533, 1987.

[12] J. Tessendorf, "Radiative transfer as a sum over paths," Physical Review A, vol. 35, 1987.

[13] J. Tessendorf, "Multiple-forward-scattering in volume rendering of participating media," 2006.

[14] C. Mätzler, "Matlab functions for Mie scattering and absorption," tech. rep., Institute of Applied Physics, University of Bern, 2002.

[15] R. G. Grainger, J. Lucas, G. E. Thomas, and G. B. L. Ewen, "Calculation of Mie derivatives," Applied Optics, vol. 43, pp. 53865393, 2004.

[16] D. W. Hahn, "Light scattering theory," tech. rep., Department of Mechanical and Aeorospace Engineering, University of Florida, 2009.

[17] T. Hauk, T. Strobl, and D. Raps, "Implementation and calibration of the icing and contamination research facility ( iCORE )," in ILASS-Europe, 25th European Conference on Liquid Atomization and Spray Systems, 2013.

[18] S. Premože, M. Ashikhmin, J. Tessendorf, R. Ramamoorthi, and S. Nayar, "Practical rendering of multiple scattering effects in participating media," in Proceedings of the Fifteenth Eurographics conference on Rendering Techniques, pp. 363-374, 2004.

[19] T. Strobl, S. Storm, D. Thompson, M. Hornung, and F. Thielecke, "Feasibility study of a hybrid ice protection system," Journal of Aircraft, 2015. 
[20] T. Strobl, R. Adam, R. M. U. Entz, and M. Hornung, "A hybrid system for ice protection and detection," in International Conference on More Electric Aircraft (MEA2015), 2015.

[21] T. Strobl, S. Storm, D. Raps, and T. Hauk, "Device and method for deicing and/or preventing ice formation and profile element and aircraft having such a device," May 14 2015. US Patent App. 14/539,491. 